\title{
Photoelectrochemical Studies of InGaN/GaN MQW Photoanodes
}

Joshua Butson $^{\mathrm{a}, \dagger}$, Parvathala Reddy Narangari ${ }^{\mathrm{a},{ }^{\dagger}, \text { }}$, Siva Krishna Karuturi ${ }^{\mathrm{a}}$, Rowena Yew $^{\mathrm{a}}$, Mykhaylo Lysevych $^{\mathrm{b}}$, Hark Hoe Tan ${ }^{\mathrm{a}}$, and Chennupati Jagadish ${ }^{\mathrm{a}}$

a Department of Electronic Materials Engineering, Research School of Physics and Engineering, The Australian National University, Canberra, ACT 2601, Australia

b Australian National Fabrication Facility, ACT Node, Research School of Physics and Engineering, The Australian National University, Canberra, ACT 2601, Australia

¥Corresponding Author: parvathala.narangari@anu.edu.au

$\dagger$ Both authors contributed equally

\begin{abstract}
The research interest in photoelectrochemical (PEC) water splitting is ever growing due to its potential to contribute towards clean and portable energy. However, the lack of low energy band gap materials with high photocorrosion resistance is the primary setback inhibiting this technology from commercialisation. The ternary alloy InGaN shows promise to meet the photoelectrode material requirements due to its high chemical stability and band gap tunability. The band gap of InGaN can be modulated from the UV to IR regions by adjusting the In concentration so as to absorb the maximum portion of the solar spectrum. This paper reports on the influence of In concentration on the PEC properties of planar and nanopillar (NP) InGaN/GaN multi-quantum well (MQW) photoanodes, where NPs were fabricated using a topdown approach. Results show that changing the In concentration, while having a minor effect on the PEC performance of planar MQWs, has an enormous impact on the PEC performance of NP MQWs, with large variations in the photocurrent density observed. Planar photoanodes containing MQWs generate marginally lower photocurrents compared to photoanodes without MQWs when illuminated with sunlight. NP MQWs with 30\% In generated the highest photocurrent density of $1.6 \mathrm{~mA} / \mathrm{cm}^{2}, 4$ times greater than that of its planar counterpart and 1.8 times greater than that of the NP photoanode with no MQWs. The InGaN/GaN MQWs also slightly influenced the onset potential of both the planar and NP photoanodes. Microphotoluminescence, diffuse reflectance spectroscopy and IPCE measurements are used to explain these results.
\end{abstract}




\section{Introduction}

Since the first photoelectrochemical (PEC) demonstration by Fujishima and Honda in 1972 [1], the PEC splitting of water into $\mathrm{H}_{2}$ and $\mathrm{O}_{2}$ has gained lot of research interest. It is a clean and renewable method for producing $\mathrm{H}_{2}$, which itself is a clean, carbon-free fuel. PEC water splitting has been investigated with a wide variety of materials such as metal oxides, silicon, III-V and II-VI semiconductors, etc. [2, 3]. However, large bandgaps in excess of $3 \mathrm{eV}$ and poor hole mobility are the main drawbacks preventing oxides from being efficient photoelectrodes [3, 4]. Si and III-V semiconductors such as GaAs and InP suffer from the photocorrosion, although they are capable of inducing highly efficient water splitting [3, 5-7]. InGaN ternary alloys are promising materials for photoelectrodes owing to band gap tunability, band edges that straddle the $\mathrm{H}_{2}$ and $\mathrm{O}_{2}$ redox potentials, larger carrier mobility and high chemical stability [8]. The band gap of InGaN can be varied from 0.7 (InN) to $3.4 \mathrm{eV}$ (GaN) by varying the In concentration, which allows absorption to be tuned from the UV to far IR regions [9]. Moreover, up to an In content of 50\%, the valence and conduction band edges of InGaN straddle both the hydrogen and oxygen evolution reaction (HER and OER) potentials, which means that it can even drive direct unassisted water splitting [10, 11]. Due respectively to its highly crystalline nature and strong ionic bonds, InGaN exhibits high carrier transport and chemical stability.

In addition to these properties, InGaN nanostructures are capable of accommodating higher In concentrations while maintaining good crystal quality of InGaN layers, given that nanostructures can undergo strain relaxation [11, 12]. Furthermore, nanostructuring enhances light-matter interactions and increases the semiconductor/electrolyte interface area while reducing carrier transfer distance to the interface, boosting the PEC performance of photoelectrodes [13-16]. Nanostructures include nanowires, nanopillars and nanopyramids and are formed using either bottom-up or top-down approaches. Molecular beam epitaxy (MBE) and metal-organic chemical vapor deposition (MOCVD) are most commonly employed for the bottom-up growth of nanowires, which are grown either catalytically or epitaxially. Many recent reports on solar water splitting with $\mathrm{InGaN} / \mathrm{GaN}$ nanowires concentrate on devices grown using MBE [11, 17-21] or MOCVD [22, 23]. However, there are very few reports on the top-down processing of InGaN/GaN nanopillar (NP) photoelectrodes for water splitting $[16,24]$. The top-down approach involves the plasma etching of patterned epitaxially-grown InGaN/GaN wafers. Almost all studies, irrespective of growth method, are focused on improving the PEC performance of InGaN/GaN photoanodes by incorporating higher In 
concentrations into either planar [25-29] or nanostructured [11, 12, 17-23] devices. As a consequence, there are no reports on the comparative studies of the influence of In concentration on PEC performance of InGaN/GaN MQWs in planar and NP photoanodes. In order to carry out these studies, both the planar and NP photoanodes require similar structural quality and In content in the InGaN layers, which can be best achieved using a top-down approach since the planar and NP photoanodes can be fabricated using the same epilayer. This is not possible with bottom-up growth methods as growth conditions are different for epilayers and nanostructures. Therefore, a top-down approach is ideal for studying the influence of In on the PEC performance of planar and NP InGaN/GaN MQW photoelectrodes as they will retain a similar crystal quality and In composition. Despite this opportunity, no previous studies have been aimed at investigating the role of InGaN/GaN MQWs in both planar and NP photoanodes. Although Benton et al. reported a large enhancement in the PEC performance of InGaN/GaN NP photoanodes fabricated using a top-down approach compared to planar photoanodes, the influence of InGaN on the PEC performance of these photoanodes was not considered [16, 24].

In this work, we report on the fabrication of InGaN/GaN MQW NPs by using a topdown approach and study the influence of In concentration on the PEC performance of both planar and NP InGaN/GaN MQW photoanodes. Diffuse reflectance and microphotoluminescence were employed to analyse the optical properties of planar and NP InGaN/GaN MQW photoanodes. The dependence of the PEC performance of these photoanodes on In concentration are explained using IPCE measurements.

\section{Experimental}

InGaN/GaN MQW epilayers with 20, 30 and 50\% In, along with a GaN reference sample, were grown using MOCVD and used to fabricate the NP photoanodes. InGaN/GaN MQW NPs were fabricated by using inductively coupled plasma reactive ion etcher (ICP-RIE) to etch randomly patterned InGaN/GaN MQW epilayers. A self-assembled random masking technique was adopted to produce a $\mathrm{SiO}_{2} / \mathrm{Ni}$ nanomask [30-32]. The nanomask was produced by first depositing a $500 \mathrm{~nm} \mathrm{SiO}_{2}$ film using plasma-enhanced chemical vapour deposition (PECVD), followed by e-beam evaporation of a $10 \mathrm{~nm}$ Ni film. Rapid thermal annealing (RTA) was performed at $850{ }^{\circ} \mathrm{C}$ for $90 \mathrm{~s}$ under an $\mathrm{Ar}$ atmosphere to convert the Ni film into Ni islands. These $\mathrm{Ni}$ islands acted as a mask while etching the $\mathrm{SiO}_{2}$ using ICP-RIE, producing the $\mathrm{SiO}_{2} / \mathrm{Ni}$ random nanomask pattern. The $\mathrm{SiO}_{2} / \mathrm{Ni}$ nanomask then served to mask the InGaN/GaN MQW epilayers during the fabrication of the NPs, also performed using ICP-RIE. For the etching of 
$\mathrm{SiO}_{2}$, ICP-RIE was maintained at room temperature under $10 \mathrm{mTorr}$ chamber pressure, with a $\mathrm{CHF}_{3}$ flow rate of $40 \mathrm{sccm}$ and ICP/RF powers of 1000/30 W. For the formation of InGaN/GaN MQW NPs, the electrode temperature was maintained at $60{ }^{\circ} \mathrm{C}$ under 4 mTorr chamber pressure, with $\mathrm{Cl}_{2} / \mathrm{Ar} / \mathrm{H}_{2}$ flow rates of 25/2/5 sccm and ICP/RF powers of 1000/200 W. After ICP-RIE, NPs were dipped in diluted HF to remove the remaining mask.

Scanning electron microscopy (SEM) was used to study the morphology of the MQW NPs after removing the mask. The optical properties of the MQWs were evaluated by performing micro-photoluminescence measurements. A $325 \mathrm{~nm}$ He-Cd laser was used to excite the samples, with the emitted light collected through a 36X (NA =0.5) objective lens and detected using a Si charge-coupled device (CCD). A UV-Vis-NIR spectrophotometer was also used to carry out diffuse reflectance measurements on the planar and NP MQWs.

$\mathrm{Ti} / \mathrm{Au}(30 / 150 \mathrm{~nm})$ layers were deposited on the planar and NP samples using e-beam evaporation, followed by RTA at $400{ }^{\circ} \mathrm{C}$ for 5 minutes under an Ar atmosphere so as to form an electrical contact to the photoanodes. PEC studies were then performed on the InGaN/GaN MQW photoanodes using a three-electrode cell and a solar simulator fitted with AM 1.5 filters, with a $1 \mathrm{M} \mathrm{NaOH}$ electrolyte, a Ag/AgCl reference electrode and a Pt counter electrode.

\section{Results and Discussion}

(a)

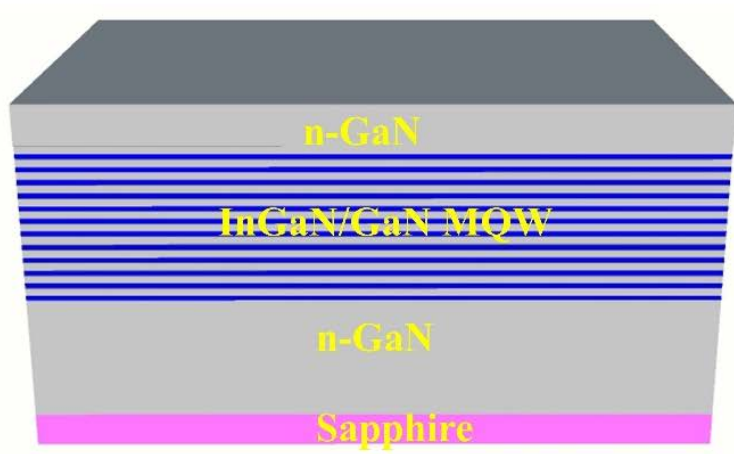

(b)

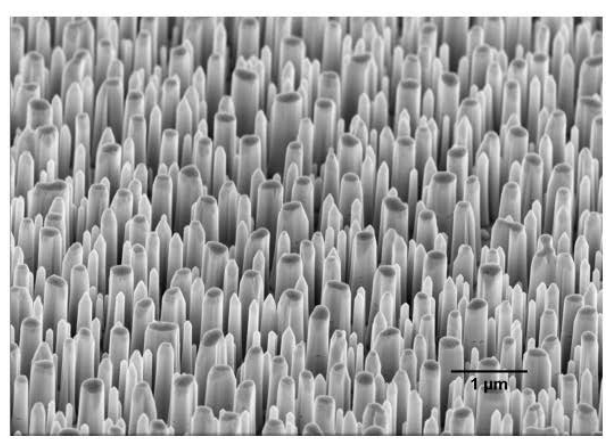

Figure 1. (a) Schematic of the InGaN/GaN MQW epilayer structure and (b) SEM image of the InGaN/GaN MQW NPs.

Figure 1a illustrates the schematic of the InGaN/GaN MQW wafers grown on sapphire using MOCVD. 10 pairs of InGaN (1.5 nm)/GaN (20 nm) MQWs were grown on $2 \mu \mathrm{m}$ of nGaN, followed by $275 \mathrm{~nm}$ of $\mathrm{n}-\mathrm{GaN}$ on top. The In concentration of the InGaN/GaN MQWs was chosen to be 20,30 and $50 \%$ and are therefore labelled as the $20 \%, 30 \%$ and $50 \%$ In 
samples respectively from here onwards, along with the GaN reference sample. The measured carrier concentration of both the top and bottom n-GaN layer was $\sim 5 \times 10^{18} \mathrm{~cm}^{-3}$. Figure $1 \mathrm{~b}$ shows the SEM image of the MQW NPs after removing the mask, fabricated using the topdown approach. The NPs maintain uniform height $(\sim 1.2 \mu \mathrm{m})$ and exhibit smooth sidewalls. The diameters of the NPs range from 60 to $250 \mathrm{~nm}$, with an average diameter of $144 \mathrm{~nm}$ as measured from the SEM image.

Diffuse reflectance measurements were carried out to investigate the influence of In concentration on the optical absorption properties of the planar and NP InGaN/GaN MQWs, the results of which are illustrated in Figure 2. As shown in Figure 2a, there is a sharp fall in diffuse reflectance for all planar samples below $370 \mathrm{~nm}$, which corresponds to the band gap of GaN. Absorption by the planar samples extends into the visible region with the incorporation of In due to the smaller band gap of InGaN. This extension of the absorption increases with In concentration, with the $30 \%$ In sample reaching up to $450 \mathrm{~nm}$. Further increasing the In concentration ( $50 \%$ In) leads to broad absorption of light in the visible region. Segregation of InN microclusters, typically associated with high In concentration, could be the reason for broad absorption in the visible region of the 50\% In sample [33]. The fringes beyond $400 \mathrm{~nm}$ are generated by Fabry-Perot interference. As shown in Figure 2b, the NP samples exhibit a significant increase in absorption within the given absorption region of the planar samples. This enhancement can be ascribed to the improved total internal reflection of the incident light as a result of the NPs $[13,30]$.

(a)

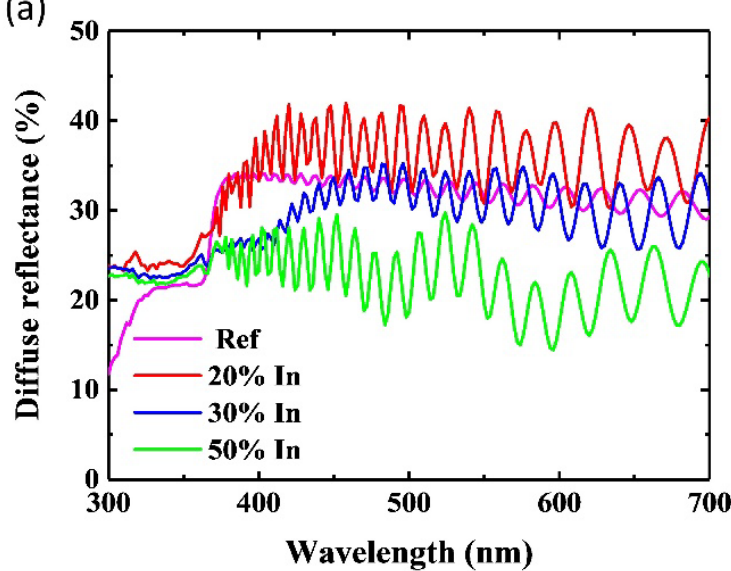

(b)

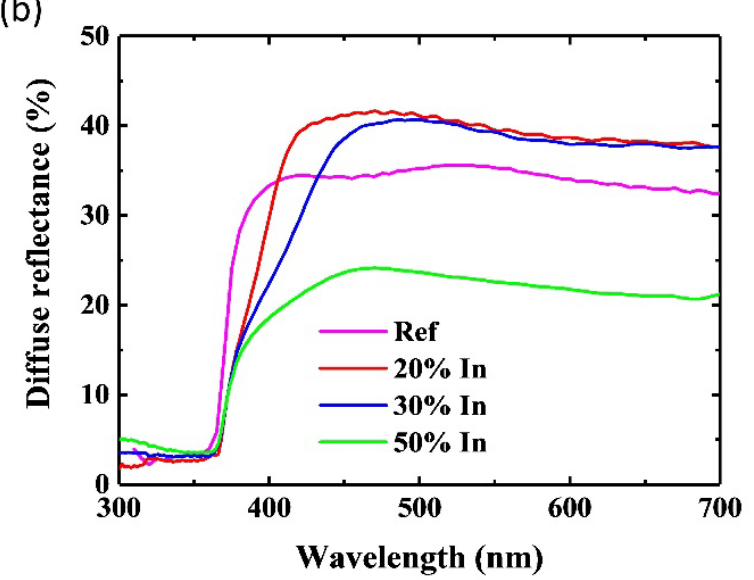

Figure 2. Diffuse reflectance spectra of (a) planar and (b) NP InGaN/GaN MQWs with varying In concentration. 
(a)

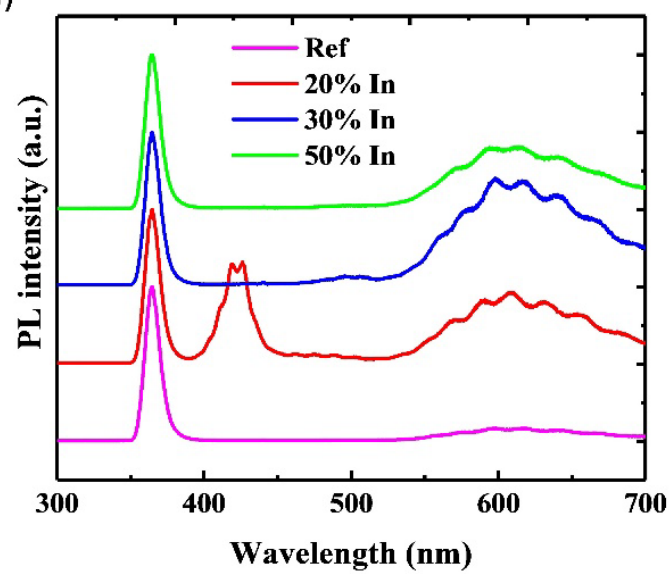

(b)

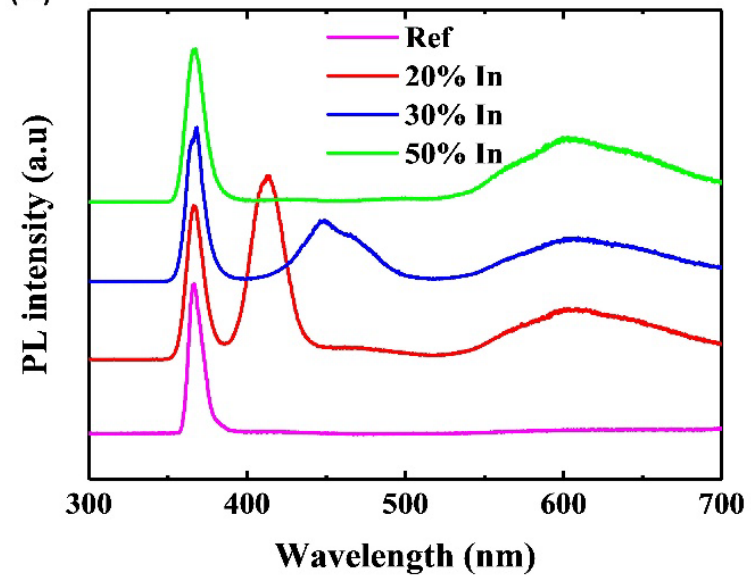

Figure 3. Room temperature photoluminescence spectra of (a) planar and (b) NP InGaN/GaN MQWs.

Photoluminescence measurements were carried out at room temperature to determine the exact band gap of the InGaN layers in the MQWs. Figure 3 shows the PL spectra of the planar and NP samples. PL intensities are normalised with respect to the peak at $365 \mathrm{~nm}$ for all samples. Both the planar and NP samples feature a strong peak at $365 \mathrm{~nm}$ corresponding to emission from bulk GaN. A broad defect-related peak, which commonly appears in MOCVDgrown GaN, is also observed at around $620 \mathrm{~nm}$ [28]. From Figure 3a, PL emissions from the planar samples that correspond to InGaN are observed only for the 20\% In sample, centred at $420 \mathrm{~nm}$, with no InGaN emissions from the $30 \%$ or $50 \%$ In samples. On the other hand, InGaN PL emissions at $\sim 450 \mathrm{~nm}$ are clearly visible from the 30\% In NP sample as shown in Figure 3b, in addition to increased emission intensity from the 20\% In NP sample compared to its planar counterpart. However, no such change is observed in the PL spectra of the $50 \%$ In NP sample. Furthermore, the InGaN PL peak of the 20\% In NPs is shifted towards shorter wavelengths by $10 \mathrm{~nm}$ compared to that of the planar structure. Strain relaxation in the NPs may be the reason behind this observed blue shift. The incorporation of high concentrations of In into InGaN creates threading dislocations and $\mathrm{InN}$ microclusters in the InGaN layer due to large lattice mismatch and miscibility gap between InN and GaN [33-35]. These defects act as non-radiative recombination centres for photo-generated carriers and thus annihilate the PL emission from InGaN. The defect density and microcluster formation in InGaN increases rapidly beyond 20\% In [34]. This could conceivably be the reason for the absence of InGaN PL peaks in the visible region of the $30 \%$ and $50 \%$ In planar samples (Figure 3a). The small footprint of the NPs helps to reduce the defect density compared to the planar structures and enhances the radiative recombination of photo-generated carriers, which results in observable 
PL emissions from the 30\% In MQW NPs. Additionally, enhanced absorption and emission by the NPs compared to the planar structures further augment the PL of the NP samples [13]. These two factors aid the surge and enrichment of the InGaN peak for the $30 \%$ and $20 \%$ In NP samples respectively (Figure 3b). However, in the case of the 50\% In NP sample, defect density and phase segregation may be above the threshold to produce any PL emissions from the MQWs. Overall, diffuse reflectance and PL spectra reveal that the $20 \%$ and $30 \%$ In samples can capture sunlight up to 420 and $450 \mathrm{~nm}$ respectively and that there is a great enhancement in absorption by the NPs compared to the corresponding planar samples.

(a)

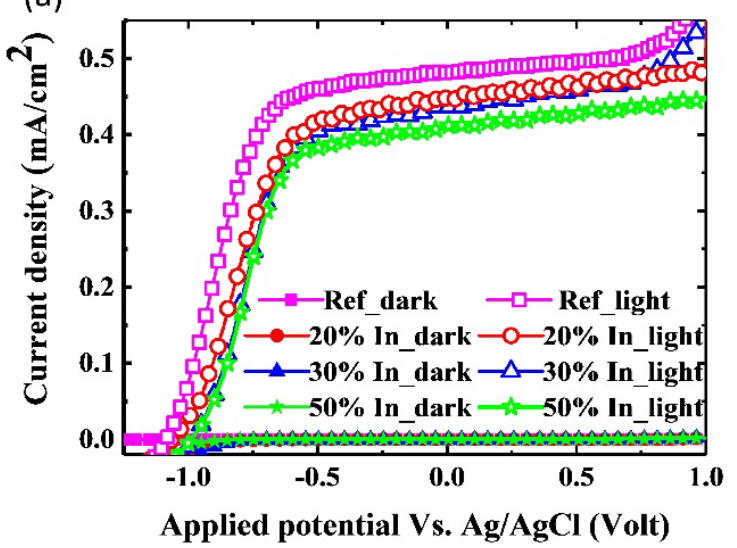

(b)

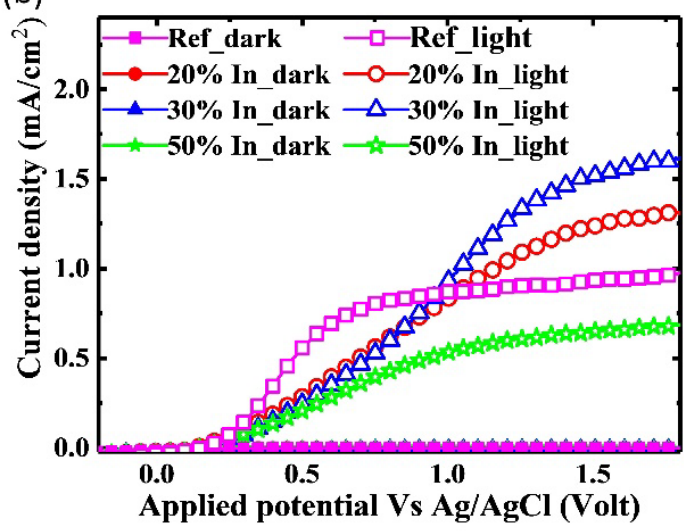

Figure 4. J-V characteristics of (a) planar and (b) NP InGaN/GaN MQW photoanodes measured under dark and sunlight illumination using a three-terminal PEC set up.

Figure 4a show the PEC performance of the planar photoanodes measured under dark and one sun illumination. All planar photoanodes generate negligible dark currents. The GaN reference photoanode generates a photocurrent density of $\sim 0.48 \mathrm{~mA} / \mathrm{cm}^{2}$, which is slightly higher than the MQW photoanodes. Photoanodes with 20\% and 30\% In show very similar saturation photocurrent densities of $\sim 0.44 \mathrm{~mA} / \mathrm{cm}^{2}$, but the $50 \%$ In photoanode witnessed a slightly lower photocurrent density of $\sim 0.4 \mathrm{~mA} / \mathrm{cm}^{2}$. A depletion layer near the semiconductor surface is responsible for the separation of photo-generated carriers, driving holes towards semiconductor/electrolyte interface and electrons into the bulk semiconductor [36]. Holes participate in the oxygen evolution reaction at the working photoanode while electrons are transferred to the counter electrode for hydrogen evolution. The depletion layer is a chargeneutral layer which is formed by band bending at the semiconductor surface. The thickness of the depletion layer depends on the carrier concentration; for the given carrier concentration of $5 \times 10^{18} \mathrm{~cm}^{-3}$, it can be less than $100 \mathrm{~nm}[30,37,38]$. The InGaN MQWs in the photoanodes are 
placed $275 \mathrm{~nm}$ (top n-GaN layer) below the semiconductor/electrolyte interface. As a result, photo-generated carriers in the InGaN MQWs cannot be separated by the depletion layer and thus do not contribute to the PEC performance of the planar MQW photoanodes. The slight drop in the photocurrent of the InGaN photoanodes can be attributed to the relatively lower crystal quality of the top n-GaN layer compared to the GaN reference photoanode. This is because the top n-GaN layer of the InGaN MQWs was grown at a lower temperature than the reference sample so as to avoid the surface diffusion of In from the InGaN MQWs. A slight positive shift in the onset potential, the potential at which photocurrent onset occurs, is observed for the MQW photoanodes compared to the GaN reference photoanode. Additionally, the anodic potential increases with In concentration for the InGaN photoanodes. The photocurrent onset occurs at $\sim-1.1 \mathrm{~V}$ for the reference photoanode and at $-1.0 \mathrm{~V}$ for the $50 \%$ photoanode. This anodic potential shift can be attributed to the depth of the quantum wells (the band alignments for InGaN alloys with varying In concentration are shown against water redox potentials in figure S1a of the supporting information). Due to energy drop of carriers in the InGaN quantum wells, additional energy is required to transfer the photo-generated carriers, which are generated in the top n-GaN layer, to the counter electrode. The amount of energy required depends on the In concentration, since well depth increases with In concentration. As a result, the onset potential increases with In concentration.

As shown in Figure 4b, the NP photoanodes exhibit vastly different J-V characteristics in comparison and generate much greater photocurrent densities. Almost 100\% improvement is observed in the photocurrent density for the GaN reference NP photoanode compared to the planar reference photoanode. The dark currents remain insignificant throughout the voltage sweep for all of the NP photoanodes, confirming that parasitic chemical reactions are not contributing to the enhanced photocurrent density. The photocurrent density generated by the NP photoanodes improves with increasing In concentration up to $30 \%$ but then falls below that of the GaN reference sample for 50\% In. For the 30\% In NP sample, the photocurrent density reaches $\sim 1.6 \mathrm{~mA} / \mathrm{cm}^{2}$, which is almost four times that of the corresponding planar photoanode and 1.8 times that of reference NP photoanode. This indicates that the InGaN MQWs play a significant role in the PEC performance of the NP photoanodes, unlike the planar photoanodes. The vast increase in semiconductor/electrolyte interface area and enhanced absorption by the NPs (Figure 3b) can be attributed to this huge improvement in the photocurrent density for NP photoanodes compared to the planar photoanodes. The NP geometry brings the InGaN MQWs in direct contact with the electrolyte and hence also into the depletion region due to the surface 
band bending occurs across the entire NP surface, which is not possible for planar photoanodes. Therefore, carriers that are photo-generated in the InGaN layers under sunlight illumination can participate in water splitting and thus contribute to the enhanced photocurrent density of the MQW NP photoanodes compared to the GaN reference NP photoanode. Due to this factor, NPs exhibit entirely different PEC characteristics compared to their planar counterparts. The photocurrent density of the InGaN NP photoanodes improves with increasing In concentration up to $30 \%$ In, which can be attributed to the absorption extended into the visible part of the spectrum by the InGaN NPs, as observed in Figure 2b and 3b. Further increasing the In concentration leads to severe defect formation which results in inferior performance of the InGaN NP photoanodes, as shown by the $50 \%$ In NP photoanodes. The stability of the planar and NP GaN reference and 30\% In photoanodes were evaluated and the results are presented in the supporting information (Figure S1b). The NP photoanodes, irrespective of In concentration, show degradation in photocurrent density with time, whereas planar photoanodes exhibit stable performance. This degradation of the NP photoanodes is attributed to the instability of m-plane $\mathrm{GaN}$ in $\mathrm{NaOH}$. For the case of planar photoanodes, the GaN cplane is exposed to the electrolyte, which is stable in $\mathrm{NaOH}$, while the long-term stability of GaN-based NP photoanodes has in fact been demonstrated using transition metal oxide cocatalysts (G. Liu et al. [32] and Benton et al. [16, 24]).

From Figure 4b, it can also be observed that the NP photoanodes exhibit higher onset and saturation potentials compared to their planar counterparts. This may be due to surface damage sustained during NP fabrication. Furthermore, similar to the planar photoanodes, there is a slight positive shift in the onset potentials of the MQW NPs with respect to the GaN reference NPs. However, this shift is not as apparent as for the planar MQWs. This may be due to dominating surface damage effects on the onset potentials of the MQW NPs.

Table 1 shows the fill factor (FF) calculated for the planar and NP photoanodes by following H. P. Wang et al. [39]. The FF for the planar photoanodes remains the same, irrespective of In concentration. This behaviour further confirms that the InGaN MQWs do not contribute to the PEC performance and that the photocurrent is generated from only the top GaN layer of the planar photoanodes. On the other hand, a significant drop in FF is noticed for the NP photoanodes. It is observed that FF decreases with increasing In concentration up to $30 \%$ and then increases for 50\% In. The drop in FF for the NP reference photoanode compared to its planar counterpart can attributed to surface damage caused during fabrication of the NPs. 
The decrease in FF with increasing In concentration can be attributed to the InGaN contribution to the PEC performance of the InGaN NP photoanodes. The improvement in FF for the $50 \%$ In NP photoanode compared to the $20 \%$ and $30 \%$ In NP photoanodes suggests that only GaN is contributing to the photocurrent due to the inferior crystal quality of the $50 \%$ In NP photoanodes.

Table 1: Fill factor calculated for the PEC performance of planar and NP photoanodes

\begin{tabular}{|c|c|c|c|}
\hline \multicolumn{2}{|c|}{ Planar Photoanodes } & \multicolumn{2}{c|}{ NP photoanodes } \\
\hline \% In & FF (\%) & \% In & FF (\%) \\
\hline Ref & 60.9 & Ref & 46.3 \\
\hline 20 & 57.6 & 20 & 23.6 \\
\hline 30 & 58.4 & 30 & 19.2 \\
\hline 50 & 59.5 & 50 & 30.0 \\
\hline
\end{tabular}

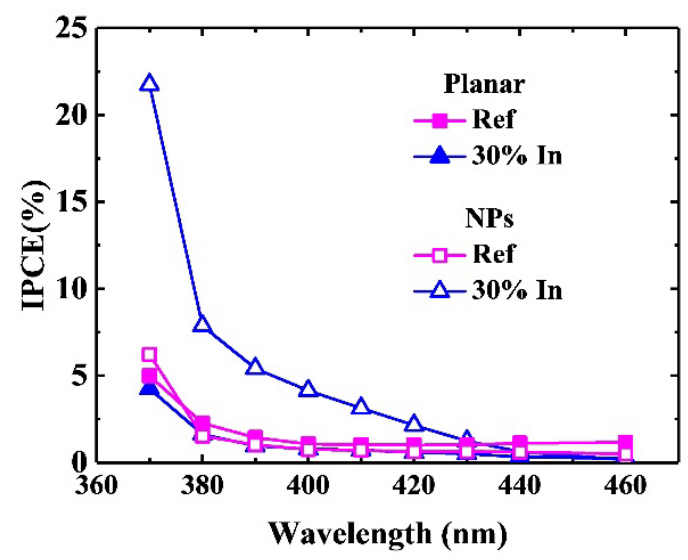

Figure 5. Incident photon-to-current efficiency (IPCE) of the GaN reference and 30\% In planar and NP photoanodes measured at zero applied potential against a $\mathrm{Ag} / \mathrm{AgCl}$ reference electrode.

To further elucidate the influence of In on the PEC performance of InGaN/GaN MQW photoanodes, the incident photon-to-current efficiency (IPCE) was measured for the GaN reference and 30\% In planar and NP photoanodes, the results of which are illustrated in Figure 5. The IPCE was determined using the following equation

$$
\operatorname{IPCE}(\%)=\frac{\left|\mathrm{J}_{\mathrm{ph}}\left(\mathrm{mA} / \mathrm{cm}^{2}\right)\right| \times 1239.8}{\mathrm{P}_{\mathrm{mono}}\left(\mathrm{mW} / \mathrm{cm}^{2}\right) \times \lambda(\mathrm{nm})} \times 100 \%
$$

where $\lambda$ is wavelength, $\mathrm{J}_{\mathrm{ph}}$ is the photocurrent density and $\mathrm{P}_{\text {mono }}$ is the incident light power at a given wavelength. The IPCE was measured at zero applied potential against a $\mathrm{Ag} / \mathrm{AgCl}$ reference electrode. As shown in Figure 5, the IPCE of each planar photoanode and the NP reference photoanode drops to negligible values beyond $370 \mathrm{~nm}$, which is below the band gap of GaN. In contrast, there is great enhancement in the IPCE for the 30\% In NP photoanodes 
near the band edge of GaN. Moreover, the IPCE of the 30\% In NP sample extends into the visible region up to $440 \mathrm{~nm}$, which is slightly below the bandgap of the MQWs observed from diffuse reflectance (Figure 2b) and PL (Figure 3b). This confirms that InGaN participates in water splitting for the NP photoanodes but does not contribute in the planar photoanodes, which agrees with observations of the planar and NP photocurrent densities (Figure 4). The IPCE confirms that InGaN plays a crucial role in the PEC performance of NP photoanodes but has little impact on the performance of planar photoanodes.

\section{Conclusions}

In conclusion, the effect of In concentration on the PEC performance of planar and NP InGaN/GaN MQW photoanodes have been investigated. The In content has little effect on the planar MQW photoanodes because the MQWs are far from the depletion layer and the semiconductor/electrolyte interface. The NP MQWs, on the other hand, generate much greater photocurrent densities that display a very strong dependence on the In content. This dependence arises from the direct exposure of the InGaN MQWs to the electrolyte, which allows holes to escape with far greater ease. By optimising the In content, the effects of absorption and carrier recombination can be balanced to achieve the greatest possible PEC performance. Out of the four NP photoanodes, including GaN reference, with In contents ranging from $30 \%$ to $50 \%$, the $30 \%$ In NPs generated the largest photocurrent density of 1.6 $\mathrm{mA} / \mathrm{cm}^{2}$, as compared to $0.44 \mathrm{~mA} / \mathrm{cm}^{2}$ generated by the corresponding planar photoanodes and $0.9 \mathrm{~mA} / \mathrm{cm}^{2}$ generated by the GaN reference NPs.

\section{Acknowledgments}

We highly acknowledge the Australian Research Council for providing financial support and the Australian National Fabrication Facility, ACT Node for providing access to epitaxial growth and fabrication facilities.

\section{References}

[1] A. Fujishima, K. Honda, Electrochemical Photolysis of Water at a Semiconductor Electrode, Nature, 238 (1972) 37-38.

[2] Y. Li, J.Z. Zhang, Hydrogen generation from photoelectrochemical water splitting based on nanomaterials, Laser \& Photonics Reviews, 4 (2010) 517-528. 
[3] S.N. Ariffin, H.N. Lim, Z.A. Talib, A. Pandikumar, N.M. Huang, Aerosol-assisted chemical vapor deposition of metal oxide thin films for photoelectrochemical water splitting, International Journal of Hydrogen Energy, 40 (2015) 2115-2131.

[4] X. Yu, T.J. Marks, A. Facchetti, Metal oxides for optoelectronic applications, Nat Mater, 15 (2016) 383-396.

[5] R. Fan, W. Dong, L. Fang, F. Zheng, M. Shen, More than 10\% efficiency and one-week stability of Si photocathodes for water splitting by manipulating the loading of the Pt catalyst and TiO2 protective layer, J. Mater. Chem. A, (2017).

[6] O. Khaselev, J.A. Turner, A Monolithic Photovoltaic-Photoelectrochemical Device for Hydrogen Production via Water Splitting, Science, 280 (1998) 425-427.

[7] M.H. Lee, K. Takei, J. Zhang, R. Kapadia, M. Zheng, Y.-Z. Chen, J. Nah, T.S. Matthews, Y.-L. Chueh, J.W. Ager, A. Javey, p-Type InP Nanopillar Photocathodes for Efficient SolarDriven Hydrogen Production, Angewandte Chemie International Edition, 51 (2012) 1076010764.

[8] J.J.T. H. P. Maruska, The preparation and properties of vapor-deposited single-crystal-line GaN, Applied Physics Letters, 15 (1969).

[9] H. Morkoc, Nitride Semiconductor Devices: Fundamentals and Applications, Wiley-VCH Verlag GmbH \& Co. KGaA, 2013.

[10] $\{$ III-V nitride semiconductors for solar hydrogen production\}, in: \{SPIE Defense, Security, and Sensing\}, SPIE, 2012, pp. \{83770B\}.

[11] M.G. Kibria, F.A. Chowdhury, S. Zhao, B. AlOtaibi, M.L. Trudeau, H. Guo, Z. Mi, Visible light-driven efficient overall water splitting using p-type metal-nitride nanowire arrays, Nature communications, 6 (2015) 6797.

[12] C. Pendyala, J.B. Jasinski, J.H. Kim, V.K. Vendra, S. Lisenkov, M. Menon, M.K. Sunkara, Nanowires as semi-rigid substrates for growth of thick, $\operatorname{In}(\mathrm{x}) \mathrm{Ga}(1-\mathrm{x}) \mathrm{N}(\mathrm{x}>0.4)$ epi-layers without phase segregation for photoelectrochemical water splitting, Nanoscale, 4 (2012) 62696275.

[13] N.P. Reddy, S. Naureen, S. Mokkapati, K. Vora, N. Shahid, F. Karouta, H.H. Tan, C. Jagadish, Enhanced luminescence from GaN nanopillar arrays fabricated using a top-down process, Nanotechnology, 27 (2016) 065304.

[14] S. Mokkapati, K.R. Catchpole, Nanophotonic light trapping in solar cells, Journal of Applied Physics, 112 (2012) 101101. 
[15] W.J. Tseng, D.H. van Dorp, R.R. Lieten, B. Mehta, P.M. Vereecken, G. Borghs, Enhanced Photocatalytic Activity of Nanoroughened GaN by Dry Etching, ECS Electrochemistry Letters, 2 (2013) H51-H53.

[16] J. Benton, J. Bai, T. Wang, Significantly enhanced performance of an InGaN/GaN nanostructure based photo-electrode for solar power hydrogen generation, Applied Physics Letters, 103 (2013) 133904.

[17] M.G. Kibria, S. Zhao, F.A. Chowdhury, Q. Wang, H.P. Nguyen, M.L. Trudeau, H. Guo, Z. Mi, Tuning the surface Fermi level on p-type gallium nitride nanowires for efficient overall water splitting, Nature communications, 5 (2014) 3825.

[18] M.G. Kibria, H.P.T. Nguyen, K. Cui, S. Zhao, D. Liu, H. Guo, M.L. Trudeau, S. Paradis, A.-R. Hakima, Z. Mi, One-step overall water splitting under visible light using multiband InGaNGaN nanowire heterostructures, ACS Nano, 7 (2013) 8.

[19] J. Kamimura, P. Bogdanoff, J. Lahnemann, C. Hauswald, L. Geelhaar, S. Fiechter, H. Riechert, Photoelectrochemical properties of $(\mathrm{In}, \mathrm{Ga}) \mathrm{N}$ nanowires for water splitting investigated by in situ electrochemical mass spectroscopy, J Am Chem Soc, 135 (2013) 1024210245.

[20] S. Fan, B. AlOtaibi, S.Y. Woo, Y. Wang, G.A. Botton, Z. Mi, High efficiency solar-tohydrogen conversion on a monolithically integrated InGaN/GaN/Si adaptive tunnel junction photocathode, Nano letters, 15 (2015) 2721-2726.

[21] B. AlOtaibi, H.P. Nguyen, S. Zhao, M.G. Kibria, S. Fan, Z. Mi, Highly stable photoelectrochemical water splitting and hydrogen generation using a double-band InGaN/GaN core/shell nanowire photoanode, Nano letters, 13 (2013) 4356-4361.

[22] L. Caccamo, J. Hartmann, C. Fabrega, S. Estrade, G. Lilienkamp, J.D. Prades, M.W. Hoffmann, J. Ledig, A. Wagner, X. Wang, L. Lopez-Conesa, F. Peiro, J.M. Rebled, H.H. Wehmann, W. Daum, H. Shen, A. Waag, Band engineered epitaxial 3D GaN-InGaN core-shell rod arrays as an advanced photoanode for visible-light-driven water splitting, ACS applied materials \& interfaces, 6 (2014) 2235-2240.

[23] M. Ebaid, J.-H. Kang, S.-H. Lim, J.-S. Ha, J.K. Lee, Y.-H. Cho, S.-W. Ryu, Enhanced solar hydrogen generation of high density, high aspect ratio, coaxial InGaN/GaN multiquantum well nanowires, Nano Energy, 12 (2015) 215-223.

[24] J. Benton, J. Bai, T. Wang, Enhancement in solar hydrogen generation efficiency using a GaN-based nanorod structure, Applied Physics Letters, 102 (2013) 173905.

[25] A. Theuwis, K. Strubbe, L.M. Depestel, W.P. Gomes, A Photoelectrochemical Study of $\operatorname{In}[$ sub x]Ga[sub 1-x]N Films, Journal of The Electrochemical Society, 149 (2002) E173. 
[26] K. Fujii, K. Kusakabe, K. Ohkawa, Photoelectrochemical Properties of InGaN for H2Generation from Aqueous Water, Japanese Journal of Applied Physics, 44 (2005) 74337435.

[27] K. Aryal, B.N. Pantha, J. Li, J.Y. Lin, H.X. Jiang, Hydrogen generation by solar water splitting using p-InGaN photoelectrochemical cells, Applied Physics Letters, 96 (2010) 052110 .

[28] W. Luo, B. Liu, Z. Li, Z. Xie, D. Chen, Z. Zou, R. Zhang, Stable response to visible light of InGaN photoelectrodes, Applied Physics Letters, 92 (2008) 262110.

[29] K. Fujii, S. Nakamura, S. Yokojima, T. Goto, T. Yao, M. Sugiyama, Y. Nakano, Photoelectrochemical Properties of InxGa1-xN/GaN Multiquantum Well Structures in Depletion Layers, The Journal of Physical Chemistry C, 115 (2011) 25165-25169.

[30] P.R. Narangari, S.K. Karuturi, M. Lysevych, H. Hoe Tan, C. Jagadish, Improved photoelectrochemical performance of GaN nanopillar photoanodes, Nanotechnology, 28 (2017) 154001.

[31] J. Zhu, L. Wang, S. Zhang, H. Wang, D. Zhao, J. Zhu, Z. Liu, D. Jiang, H. Yang, The fabrication of GaN-based nanopillar light-emitting diodes, Journal of Applied Physics, 108 (2010) 074302.

[32] G. Liu, S.K. Karuturi, A.N. Simonov, M. Fekete, H. Chen, N. Nasiri, N.H. Le, P. Reddy Narangari, M. Lysevych, T.R. Gengenbach, A. Lowe, H.H. Tan, C. Jagadish, L. Spiccia, A. Tricoli, Robust Sub-Monolayers of Co3O4Nano-Islands: A Highly Transparent Morphology for Efficient Water Oxidation Catalysis, Advanced Energy Materials, 6 (2016).

[33] D. Holec, Y. Zhang, D.V.S. Rao, M.J. Kappers, C. McAleese, C.J. Humphreys, Equilibrium critical thickness for misfit dislocations in III-nitrides, Journal of Applied Physics, 104 (2008).

[34] X.-m. Cai, S.-w. Zeng, B.-p. Zhang, Fabrication and characterization of InGaN p-i-n homojunction solar cell, Applied Physics Letters, 95 (2009) 173504.

[35] I.h. Ho, G.B. Stringfellow, Solid phase immiscibility in GaInN, Applied Physics Letters, 69 (1996) 2701-2703.

[36] B. AlOtaibi, M. Harati, S. Fan, S. Zhao, H.P. Nguyen, M.G. Kibria, Z. Mi, High efficiency photoelectrochemical water splitting and hydrogen generation using GaN nanowire photoelectrode, Nanotechnology, 24 (2013) 175401.

[37] R. Calarco, M. Marso, T. Richter, A.I. Aykanat, R. Meijers, V.D.H. A, T. Stoica, H. Luth, Size-dependent photoconductivity in MBE-grown GaN-nanowires, Nano letters, 5 (2005) 981984. 
[38] T. Richter, H.L. Meijers, R. Calarco, M. Marso, Doping concentration of GaN nanowires determined by opto-electrical measurements, Nano letters, 8 (2008) 3056-3059.

[39] H.P. Wang, K. Sun, S.Y. Noh, A. Kargar, M.L. Tsai, M.Y. Huang, D. Wang, J.H. He, High-Performance a-Si/c-Si Heterojunction Photoelectrodes for Photoelectrochemical Oxygen and Hydrogen Evolution, Nano letters, 15 (2015) 2817-2824. 


\section{Supporting Information}

\section{Photoelectrochemical Studies of InGaN/GaN MQW Photoanodes}

Joshua Butson $^{\mathrm{a}, \dagger}$, Parvathala Reddy Narangari ${ }^{\mathrm{a}, \dagger, \ddagger}$, Siva Krishna Karuturi ${ }^{\mathrm{a}}$, Rowena Yew ${ }^{\mathrm{a}}$, Mykhaylo Lysevych ${ }^{\mathrm{b}}$, Hark Hoe Tan ${ }^{\mathrm{a}}$, and Chennupati Jagadish ${ }^{\mathrm{a}}$

a Department of Electronic Materials Engineering, Research School of Physics and Engineering, The Australian National University, Canberra, ACT 2601, Australia

b Australian National Fabrication Facility, ACT Node, Research School of Physics and Engineering, The Australian National University, Canberra, ACT 2601, Australia

†Corresponding Author: parvathala.narangari@anu.edu.au

$\dagger$ Both authors contributed equally

(a)

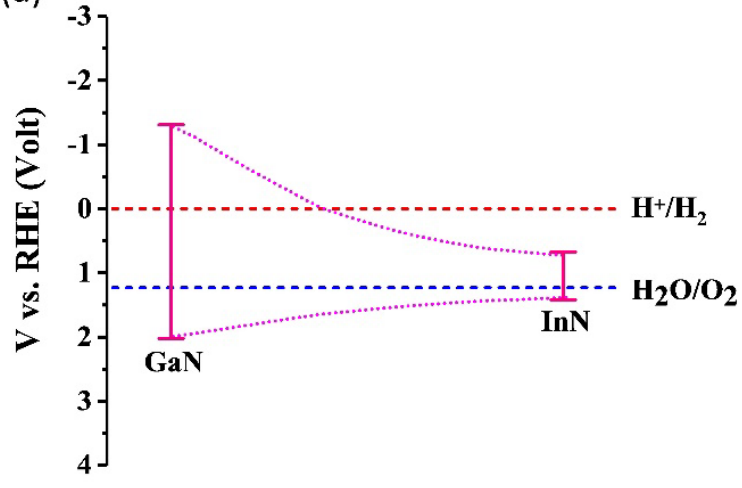

(b)

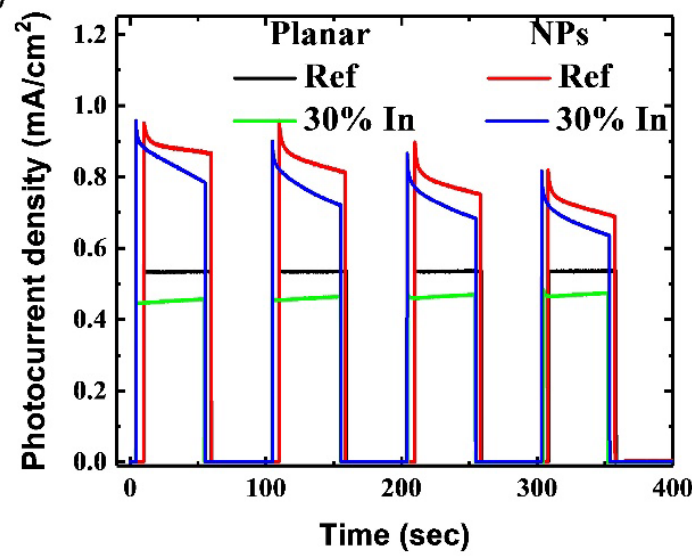

Figure S1: (a) Schematic of InGaN alloy band alignment with respect to the water redox potential with varying In concentration and (b) photo-amperometry J-t measurement for reference and 30\% In planar and NP photoanodes. 